\title{
Correspondence
}

Cerebrovasc Dis 2013;36:418-419

DOI: $10.1159 / 000356484$

\section{Neuropathology of Cortical Superficial Siderosis and Cerebral Amyloid Angiopathy: New Insights, New Questions}

\section{Andreas Charidimou, David J. Werring}

Stroke Research Group, UCL Institute of Neurology and National Hospital for Neurology and Neurosurgery, London, UK

Cortical superficial siderosis (cSS) is detected as a characteristic curvilinear ('gyriform') pattern of low signal on blood-sensitive MRI sequences, preferentially affecting the cerebral convexities [1] and reflecting blood residues in the superficial (subpial) layers of the cerebral cortex or in the subarachnoid space. cSS has emerged as a promising characteristic neuroimaging marker of sporadic cerebral amyloid angiopathy (CAA) [2]: one study reported cSS in $60.5 \%$ ( $\mathrm{n}=38$; mean age: $70 \pm 6.4$ years) of patients with histopathologically proven CAA, compared with no control subjects with proven non-CAA intracerebral haemorrhage $(\mathrm{n}=22$; mean age: $54 \pm 18$ years) [3]. In another recent study we found cSS in $40 \%$ of patients with a clinical-radiological diagnosis of CAA, compared with 5\% in non-CAA-related intracerebral haemorrhage [4].

In this issue of Cerebrovascular Diseases, De Reuck et al. [5] report new data on the neuropathological correlates of cSS from a 7 -tesla post-mortem MRI study. Of the 120 post-mortem brains of elderly subjects with various neurodegenerative and cerebrovascular pathologies, cSS on T2*-weighted MRI was found in 45 cases (15 with CAA and 30 without 'significant CAA'). A total of 83 separate 'zones' of cSS were detected: 25 areas of disseminated cSS (affecting more than 3 sulci) and 58 areas of focal cSS (affecting less than 3 sulci). Histopathologically, zones of disseminated cSS were spatially associated with lobar haematomas $(\mathrm{n}=14)$ and cerebral infarcts $(n=11)$. By contrast, zones of focal cSS were spatially associated with cerebral microbleeds $(\mathrm{n}=19)$ and cerebral microinfarcts $(n=39)$. Disseminated cSS was more commonly associated with an old lobar haematoma in the CAA group than in the nonCAA group (53 vs. $3 \%$; $p=0.003$ ). Somewhat unexpectedly, focal cSS was spatially associated with a microbleed in $4 \%$ of the CAA cases compared with $31 \%$ of the non-CAA cases $(p=0.03)$, and with cortical microinfarcts in 32 versus $53 \%(\mathrm{p}=0.25)$. The authors concluded that cSS is related to both haemorrhagic and ischaemic lesions and advised caution in interpreting cSS as another haemorrhagic manifestation of CAA.

This study adds new pathological insights to our current knowledge of cSS. However, some potential limitations need to be considered in interpreting the findings. First, there may be a selection bias in an autopsy study towards severely affected or atypical cases, which might limit generalisability to other populations. Second, the relevance of cSS detected by ex vivo high-field MRI (with high sensitivity) compared with clinical in vivo scans at lower field strengths is unclear. Third, the 'zones' of cSS detected in the limited brain sections examined could introduce a sampling bias as cSS distribution might not be uniform across the brain. Finally, sampling bias also makes it likely that some autopsy cases classified as non-CAA might have some degree of CAA pathology, a very common finding in the elderly. Moreover, 'significant CAA' was defined as a majority of vessels positive for amyloid- $\beta$ in at least 3 out of 4 samples examined; those cases with few amyloid- $\beta$-stained vessels in 1 or 2 samples were categorised as 'not-CAA', but they may harbour some CAA pathology. This could contribute to cSS in putative 'non-CAA' cases, and might explain the association of cSS with lobar microbleeds (a known marker of CAA) in this group. Notwithstanding these limitations, the study shows that cSS was significantly more common in the CAA than in the non-CAA group: $15 / 22$ (68.2\%; 95\% CI: 45.1-86.1) in CAA brains versus 30/98 (30.6\%; 95\% CI: 21.7-40.7) in non-CAA brains ( $\mathrm{p}=0.001$, using a simple $\chi^{2}$ test); CAA brains also had a higher prevalence of disseminated cSS than non-CAA brains $(\mathrm{p}=0.01)$, which is in agreement with a recent clinical-radiological study [4].

Although this study is an important first step in investigating the neuropathological associations of cSS, the cross-sectional study design provides only a snapshot of dynamic small vessel disease processes. cSS in CAA could be due to several mechanisms, including repeated haemorrhage from brittle superficial cortical or leptomeningeal vessels into the adjacent subarachnoid space or the periadventitial compartment, or leakage from a previous lobar intracerebral haemorrhage or microbleed into the subarachnoid space. De Reuck et al. [5] hypothesise that, in addition, cSS could result from cortical microinfarcts (perhaps through haemorrhagic transformation). The finding of these other lesions in close vicinity to cSS cannot prove the direction of causality. For example, recent data strongly suggest that cSS may in some cases precede lobar haemorrhage $[3,6-8]$. cSS may also reflect focally active severe microangiopathy, increasing the risk of both microinfarcts and microbleeds. Indeed, although the numbers were small, there was a trend for higher lesion burden (including cortical microinfarcts and microbleeds) in the CAA-cSS group compared with the CAA group without cSS in the current study.

The findings of De Reuck et al. [5] remind us of the need to think about small vessel disease damage and CAA as a continuum of 'haemorrhagic' and 'ischaemic' microangiopathic processes [9]. Further studies are needed to validate their findings and interpretation. More intensive neuropathological studies, with post-mortem imaging of the whole brain, will be valuable. Serial MRI studies (ideally at high field strengths) will also help to unravel the exact sequence of events and mechanisms linking CAA, cSS and other manifestations of small vessel disease [10].

\section{KARGER}

E-Mail karger@karger.com

www.karger.com/ced
C 2013 S. Karger AG, Basel

$1015-9770 / 13 / 0366-0418 \$ 38.00 / 0$
Dr. Andreas Charidimou

National Hospital for Neurology and Neurosurgery

Box 6, Queen Square

London WC1N 3BG (UK)

E-Mail andreas.charidimou.09@ucl.ac.uk 


\section{References}

1 Linn J, Herms J, Dichgans M, Brückmann H, Fesl G, Freilinger T, Wiesmann M: Subarachnoid hemosiderosis and superficial cortical hemosiderosis in cerebral amyloid angiopathy. AJNR Am J Neuroradiol 2008;29: 184-186.

2 Charidimou A, Gang Q, Werring DJ: Sporadic cerebral amyloid angiopathy revisited: recent insights into pathophysiology and clinical spectrum. J Neurol Neurosurg Psychiatry 2012;83:124-137.

-3 Linn J, Halpin A, Demaerel P, Ruhland J, Giese AD, Dichgans M, van Buchem MA, Brückmann H, Greenberg SM: Prevalence of superficial siderosis in patients with cerebral amyloid angiopathy. Neurology 2010; 74:1346-1350.

4 Charidimou A, Jäger RH, Fox Z, Peeters A, Vandermeeren Y, Laloux P, Baron JC, Werring DJ: Prevalence and mechanisms of cortical superficial siderosis in cerebral amyloid angiopathy. Neurology 2013;81:626-632.

$\checkmark 5$ De Reuck J, Deramecourt V, Cordonnier C, Auger F, Durieux N, Pasquier F, Bordet R, Defebvre L, Caparros-Lefebvre D, Maurage CA, Leys D: Superficial siderosis of the central nervous system: a post-mortem 7.0-tesla magnetic resonance imaging study with neuropathological correlates. Cerebrovasc Dis 2013;36:412-417.
6 Linn J, Wollenweber FA, Lummel N, Bochmann K, Pfefferkorn T, Gschwendtner A, Brückmann H, Dichgans M, Opherk C: Superficial siderosis is a warning sign for future intracranial hemorrhage. J Neurol 2013;260:176-181.

7 Charidimou A, Peeters A, Jäger HR, Fox Z, Vandermeeren Y, Laloux P, Baron JC, Werring DJ: Cortical superficial siderosis and intracerebral hemorrhage risk in cerebral amyloid angiopathy. Neurology 2013, E-pub ahead of print.

8 Takeda S, Yamazaki K, Miyakawa T, Onda K, Hinokuma K, Ikuta F, Arai $\mathrm{H}$ : Subcortical hematoma caused by cerebral amyloid angiopathy: does the first evidence of hemorrhage occur in the subarachnoid space? Neuropathology 2003;23:254-261.

-9 Pantoni L: Cerebral small vessel disease: from pathogenesis and clinical characteristics to therapeutic challenges. Lancet Neurol 2010;9:689-701. 10 Charidimou A, Baron JC, Werring DJ: Transient focal neurological episodes, cerebral amyloid angiopathy, and intracerebral hemorrhage risk: looking beyond TIAs. Int J Stroke 2013;8:105-108. 